\title{
Automatic Relevance Determination for Identifying Thalamic Regions Implicated in Schizophrenia
}

\author{
Antony Browne, Angela Jakary, Sophia Vinogradov, Yu Fu, and Raymond F. Deicken
}

\begin{abstract}
There have been many theories about and computational models of the schizophrenic disease state. Brain imaging techniques have suggested that abnormalities of the thalamus may contribute to the pathophysiology of schizophrenia. Several studies have found the thalamus to be altered in schizophrenia, and the thalamus has connections with other brain structures implicated in the disorder. This paper describes an experiment examining thalamic levels of the metabolite $\mathrm{N}$-acetylaspartate (NAA), taken from schizophrenics and controls using in vivo proton magnetic resonance spectroscopic imaging. Automatic relevance determination was performed on neural networks trained on this data, identifying NAA group differences in the pulvinar and mediodorsal nucleus, underscoring the importance of examining thalamic subregions in schizophrenia.
\end{abstract}

\section{INTRODUCTION}

$\mathbf{S}$ CHIZOPHRENIA is one of the most disabling and persistent mental illnesses, often striking in the youth of the sufferer, and having potentially severe effects on the sufferer's family or other caregivers. The disease has huge effects on the quality of life, employment, and productivity of sufferers. Research over the last three decades has led to a wide variety of findings on the potential causes of schizophrenia, including genetic associations, neurochemical abnormalities, neuroanatomical deficits as revealed by neuroimaging procedures, cognitive deficits, and many environmental and social factors that may be implicated in the disease. However, there is no single cohesive theoretical framework joining genes, biochemistry, cognition, and psychological outcome, i.e., a framework for integrating the biological and psychological aspects of the disease. Although the introduction of atypical antipsychotic drugs has eliminated many of the serious sedative and motor side effects seen with the older antipsychotics, the main focus of clinical treatment still remains control of psychotic symptoms. There are no methods of preventing this debilitating illness from developing. Conventional therapies have not targeted the cognitive deficits that appear to be significant in schizophrenia and that are correlated with neuroimaging findings. Therefore, a challenge exists to provide a model for understanding schizophrenia that

Manuscript received September 26, 2006; revised May 4, 2007 and July 20, 2007; accepted August 28, 2007. This work was supported in part by a Stanley Foundation Research Award.

A. Browne and Y. Fu are with the Computing Department, School of Engineering and Physical Sciences, University of Surrey, Guildford, Surrey GU2 7XH, U.K. (e-mail: a.browne@ surrey.ac.uk).

A. Jakary is with the Veterans Affairs Medical Center, Psychiatry Service, San Francisco, CA 94121-1598 USA.

R. F. Deicken and S. Vinogradov are with the Veterans Affairs Medical Center, Psychiatry Service, San Francisco, CA 94121-1598 USA and also with the Department of Psychiatry, University of California, San Francisco, CA 94143 USA

Digital Object Identifier 10.1109/TNN.2008.2000203

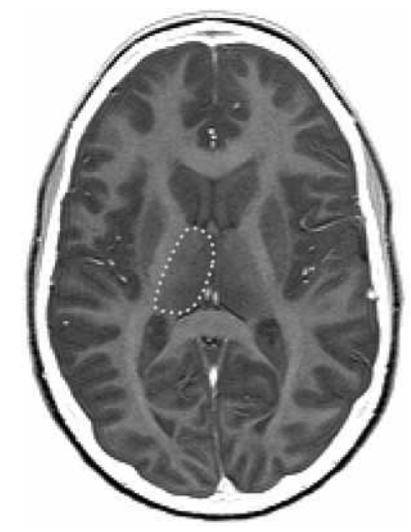

Fig. 1. Axial view of the two lobes of the thalamus, situated in the center of the image and outlined with a broken line.

will eventually provide insights to be used in therapy, moving beyond mere symptomatic control [1].

\section{A. Neuroimaging Studies of the Thalamus}

Neuroimaging research into the etiology of schizophrenia over the last three decades has identified regional brain abnormalities implicated in the neurocognitive and behavioral symptomatology of this devastating brain disease. One of the emerging key brain structures that participates in neural circuits hypothesized to be dysfunctional in schizophrenia is the thalamus. An indication of the position of the thalamus in the brain is given in Fig. 1. In this figure [taken from an magnetic resonance (MR) image, with the front of the head to the top of the page], the left thalamus is surrounded by the broken line (the right thalamus can be seen adjacent to it). The thalamus has traditionally been considered as a relay center for afferent sensory pathways, regulating and transmitting peripheral stimulation to areas of the cortex. However, it also plays a crucial role in memory, executive functioning and attention. For example, gray matter density in the thalamus is positively correlated with IQ in healthy young people [2].

Neuroimaging studies have demonstrated somewhat inconsistent thalamic alterations in schizophrenia, including changes in thalamic volume [3] and connectivity [4] as shown by MRI; abnormalities in glucose metabolism in the mediodorsal, anterior, and pulvinar regions of the thalamus as measured by blood-oxygen-level-dependent (BOLD) functional magnetic resonance imaging (fMRI) [5]-[9]; differences in thalamic mean diffusivity as revealed by MRI diffusion tensor imaging [10]; reductions in volume and/or neuronal number in the mediodorsal nucleus and pulvinar [11]-[16]; and altered glutamatergic N-methyl-D-aspartic acid (NMDA) receptor 
expression [17] and synaptic dysfunction or loss [18] in the anterior and mediodorsal thalamic nuclei in patients with schizophrenia. In our work, we wanted to generate in vivo experiments to explore the relationship between different nuclei in the thalamus and schizophrenia. An amino acid currently utilized as an indicator of neuronal, axonal, and dendritic integrity is $\mathrm{N}$-acetylaspartate (NAA), the levels of which can be detected by proton magnetic resonance spectroscopy (1H MRS) (a useful tool for the in vivo quantification of brain metabolites). NAA's exact biological function has not been precisely elucidated. Evidence suggests that it is involved in the transport of water out of neurons [19] and may also be important in forming the myelin sheath around axons [20]. NAA concentrations have been shown to diminish with axonal loss, and there is evidence linking NAA to compromised functional and/or structural neuronal integrity of the (large number of) neurons in a voxel in a wide range of disorders [21], [22]. Combining $1 \mathrm{H}$ MRS and measures of cognitive ability has highlighted the relationship of NAA to neuropsychological functioning in normal human brains. For example, Jung et al. [23], [24] have reported a correlation between occipitoparietal white matter NAA levels and IQ in young adults. NAA concentrations in the thalamus may also reveal key neuroanatomical alterations in schizophrenia. Recent studies comparing schizophrenics with controls have noted: 1) reduced thalamic NAA levels in schizophrenia [26]-[28]; and 2) no group differences in NAA levels [29]-[31]. Discrepancies are thought to be due to several factors such as varying voxel size, position, and slice thickness; averaging many voxels over a large region; and voxel tissue heterogeneity. Studies with more localized voxel placement have detected reduced bilateral mediodorsal and anterior thalamic NAA levels in schizophrenia [26]-[28]. However, these studies did not examine additional thalamic subregions. In this study, we examined schizophrenia-related subregional thalamic NAA abnormalities using neural network techniques. This involved training multilayer perceptrons (MLPs) using NAA values recorded from different areas of the thalamus as inputs and the classification of schizophrenic versus control as a target output.

\section{B. Inferring the Importance of Input Features Using Neural Networks}

Neural networks are no longer "black boxes"; techniques exist to extract knowledge that they have learned when modeling the task they have been applied to (for example, rules/decision trees can be extracted-see [32]). However, in this research, we were not interested in extracting knowledge in the form of rules/decision trees, but in locating areas of the thalamus implicated in schizophrenia by inferring the importance of input features from the spectroscopic data. Inferring the relative importance of features has received a great deal of attention in the machine learning and statistics research communities [33]. A successful Bayesian method for assessing the importance of features is automatic relevance determination (ARD), which can be applied to standard feedforward neural networks [34], [35] and has many applications [36], [37]. This approach optimizes the model evidence (marginal likelihood), the classic criterion for Bayesian modeling, and generates hyperparameters $\alpha$, explicitly representing the relevance of different input features. A separate hyperparameter $\alpha_{i}$ is associated with each input variable $I_{i}$ of an MLP. Each of these $\alpha_{i}$ represents the inverse variance of the distribution of the weights fanning out from a particular input. During training, these hyperparameters are modified. The initial prior distribution $P\left(w_{i}\right)$ of the weights $w_{i}$ is assumed to have a Gaussian distribution with zero mean which is in the form

$$
P\left(w_{i}\right)=\frac{1}{Z_{w}\left(\alpha_{i}\right)} \exp \left(-\frac{\alpha_{i}}{2}\left\|w_{i}\right\|^{2}\right)
$$

where $w_{i}$ are the weights fanning out from the input $I_{i}, Z_{w}\left(\alpha_{i}\right)$ is the normalization constant, and $\alpha_{i}$ is the hyperparameter of weights $w_{i}$. As training of the MLP progresses, every hyperparameter $\alpha_{i}$ is reestimated to a new value $\alpha_{i}^{\text {new }}$ using [34]

$$
\alpha_{i}^{\text {new }}=\frac{\gamma_{i}}{\mu_{i}^{2}}
$$

where $\mu_{i}$ is the posterior mean of the weights corresponding to an input $i$ and $\gamma_{i} \in[0,1]$ is a measure of how "well determined" its corresponding parameter $w_{i}$ is by the data [34]. The quantity $\gamma$ is calculated from the eigenvalues $\lambda_{i}$ of the matrix $W$ of weights and biases in the network using

$$
\gamma \equiv \sum_{i=1}^{W} \frac{\lambda_{i}}{\lambda_{i}+\alpha} .
$$

The quantity $\gamma$ effectively captures the influence of the likelihood and the prior (i.e., when $\gamma_{i} \approx 1, \alpha_{i}$ is small and $w_{i}$ fits the data; when $\gamma_{i} \approx 0, \alpha_{i}$ is large and $w_{i}$ is highly constrained by the prior). An excellent explanation of this procedure is available [38] and a useful software implementation and associated textbook can be found [39], [40]. On completion of training, a small $\alpha_{i}$ means that the corresponding input (e.g., area of the thalamus) is important in schizophrenia. Conversely, large $\alpha_{i}$ indicate that the regions of the thalamus associated with that input are not important in schizophrenia.

\section{METHOD}

All 18 subjects were male (in order to remove confounding effects from differences between male and female brains). All nine schizophrenic subjects met the Diagnostic and Statistical Manual of Mental Disorders (DSM-IV) criteria for schizophrenia, and had no history of head injury, organic mental disorder, neurological disorder, cerebrovascular disease, major mood disorder, or anxiety disorder. They were also free of any clinically significant alcohol/substance abuse in the 12 months prior to the study. The nine control subjects had no history of substantial medical illness, head injury, neurological or psychiatric disorder, or clinically significant alcohol/substance abuse. There was no significant difference between patients and controls in age, and all subjects were right-handed. All MRI/MRS studies were performed on a 1.5-T Magnetom VISION system using a standard circularly polarized head coil. 
MRI sequences included axial whole brain T2-weighted images from double spin echo (DSE) (TR/TE1/TE2 $=3000 / 20 / 80$ $\mathrm{ms}$, resolution $=1 \times 1.4 \mathrm{~mm}^{2}, 3-\mathrm{mm}$ slice thickness, oriented $-10^{\circ}$ off the planum sphenoidale); and coronal whole brain T1-weighted images from 3-D magnetization-prepared rapid gradient echo (MP-RAGE) $(\mathrm{TR} / \mathrm{T} 1 / \mathrm{TE}=10 / 250 / 4 \mathrm{~ms}$, resolution $=1 \times 1 \mathrm{~mm}^{2}, 1.4-\mathrm{mm}$ slice thickness, oriented perpendicular to the DSE). The MRI scans of both groups were examined by a neuroradiologist and determined to be free of abnormalities. Multislice $1 \mathrm{H}$ magnetic resonance spectroscopic imaging (MRSI) (TR/TE $=1960 / 135 \mathrm{~ms}$ ) was performed using three axially oblique $15-\mathrm{mm}$-thick sections oriented to the DSE angle, with the middle slice centered on the thalamus. The nominal voxel size was $0.9 \mathrm{~mL}$, and the effective voxel size (after reconstruction of MRSI data volumes) was $1.5 \mathrm{~mL}$. Each subject was assigned a numerical code for blind measurement and data processing. The MRI tissue segmentation and volumetric techniques described in [41] were used to segment the brain into cortical and subcortical gray matter, sulcal and ventricular cerebrospinal fluid (CSF), white matter, and white matter signal hyperintensities. The boundaries of the thalamus were manually delineated on each DSE axial slice containing visible thalamus to create thalamic tissue volume masks. In-house software was then used to coregister the segmentation and thalamic volume information with the MRSI data, to obtain the tissue composition of each MRSI voxel so that the NAA concentrations could be tissue corrected.

The data set containing the NAA values initially proved problematic for training an MLP, for a number of reasons. The number of voxels containing thalamic tissue (containing varying amounts of thalamic tissue from $1 \%$ to $100 \%$ ) for each subject varied (from 80 to 100 voxels between subjects), and the potential number of input parameters would cause tremendous overfitting problems for a neural network (together with extended training times). In addition, there was little correspondence of the $X$ and $Y$ coordinates of voxels between subjects, because different subjects' brains have different sizes and shapes, and brain positioning within the scanner varies between subjects. To counter these problems, the $X$ and $Y$ coordinates were scaled to total $X$ and $Y$ ranges per subject, and suitable $X$ and $Y$ quantization values were chosen so that voxel NAA concentrations could be compared across subjects, taking into account the percentage of thalamic tissue in each voxel. The result of this was a set of 20 mean thalamic NAA concentration values for each subject, distributed across a 2-D grid representing an "average" thalamus (scaled across all subjects). Using these 20 NAA concentrations as inputs and the classes $1=$ schizophrenic and $0=$ control as target outputs, MLPs were constructed and then trained using the scaled conjugate gradients algorithm [42] and Bayesian weight regularization [34], [35] to prevent overfitting. Once the networks were trained, ARD parameters were generated for each input. The classification performance of the MLPs was evaluated using leave-one-out cross validation. A range of hidden unit values from one to 20 was evaluated, with 100 MLPs being trained for each hidden unit setting using different random initial weights and weight ranges for each (to compensate for
TABLE I

LEAVE-ONE-OUT CROSS-VALIDATION PERFoRMANCE OF THE MLP AND STATISTICAL TECHNIQUES

\begin{tabular}{|l|r|}
\hline Technique & Percentage correct \\
\hline \hline Linear regression & 78 \\
\hline Logistic regression & 89 \\
\hline MLP & 100 \\
\hline
\end{tabular}

spurious results being caused by the small number of items in the training set). For each model trained, records were made of classification performance and $\alpha$ values generated by the ARD algorithm, and classification performance/ARD values were averaged over all 100 runs of each model. As a comparison, the MLPs performance was compared with that of two standard statistical techniques (multiple step linear regression and logistic regression, both using leave-one-out cross validation).

\section{RESULTS}

An MLP with a minimum of six hidden units, using the NAA values as input, could reliably differentiate schizophrenic subjects from controls for all subjects $(18 / 18=100 \%$ accuracy $)$. As a comparison, the two standard statistical techniques could perform the same task with reduced accuracy (see Table I). This implied that the MLP was producing a more accurate model of the data than the other two techniques, and should be the model selected when attempting to understand the influence of particular input features (i.e., NAA level in a particular area of the thalamus) and the schizophrenic disease state.

A schematic diagram of the thalamus, with most of the nuclei unlabeled and only those associated with interesting results in this study, is shown in Fig. 2. Fig. 3 shows a contour graph of ARD values for the grid of $X Y$ coordinates across the thalamus. Here, light colors show areas where ARD has identified regions of importance for differentiating schizophrenics from controls, whereas dark areas indicate regions unimportant for discriminating these groups. Figs. 2 and 3 are superimposed in Fig. 4, which shows that ARD has highlighted the right mediodorsal nucleus and pulvinar of the thalamus as being important in schizophrenia. In addition, there is a lighter patch on the top right of this figure, corresponding to the left anterior thalamus. After inspecting of many of these regions of the thalamus, schizophrenic subjects have markedly lower NAA concentrations than controls. However, it is not possible to separate the schizophrenic and control groups by simply inspecting NAA values at specific $X Y$ coordinates.

\section{DISCUSSIONS AND CONCLUSION}

Using a nonlinear neural network such as the Bayesian MLP for modeling a psychiatric data set provides enhanced classification performance when compared to two standard statistical techniques. However, the current model used a small number of inputs, and there are questions on the scalability of this Bayesian MLP-based technique to a data set containing many more input voxels (particularly in terms of extended training times and generalization ability when there are a large number of voxels per subject, but few subjects). Recent work on full-brain analysis 


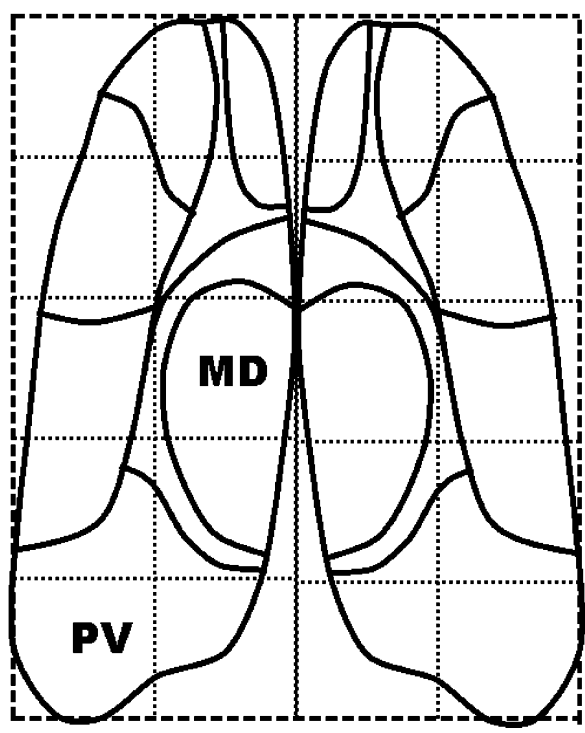

Fig. 2. Schematic diagram of thalamus, indicating mediodorsal nucleus (MD) and pulvinar (PV).

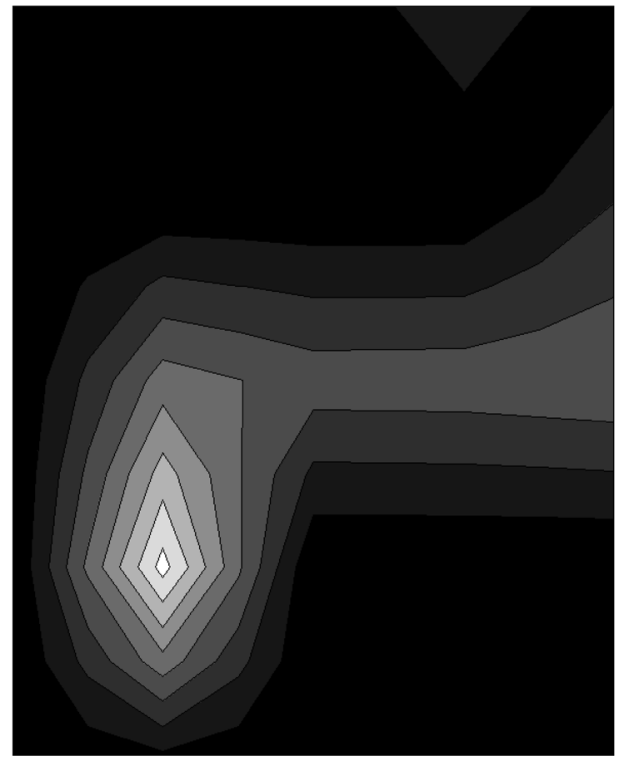

Fig. 3. Contour graph of ARD results for thalamus. Light shading includes low (important) ARD values.

having 40-K voxels as inputs [43] has demonstrated that support vector machines (SVMs) [44] and cross validation can be used in these circumstances. In addition, other work has shown that ARD can be performed on SVMs [45], [46]. An alternative technique is to use Bayesian MLPs on a subset of the available voxels that has been selected empirically using logistic regression [47].

Training neural networks with ARD has highlighted particular areas of the thalamus, such as the right mediodorsal and pulvinar nuclei (with lesser involvement of the left anterior nucleus) as being important in schizophrenia. These findings support previous research indicating structural and functional alterations in these areas of the thalamus in schizophrenia. For example, functional neuroimaging studies have found

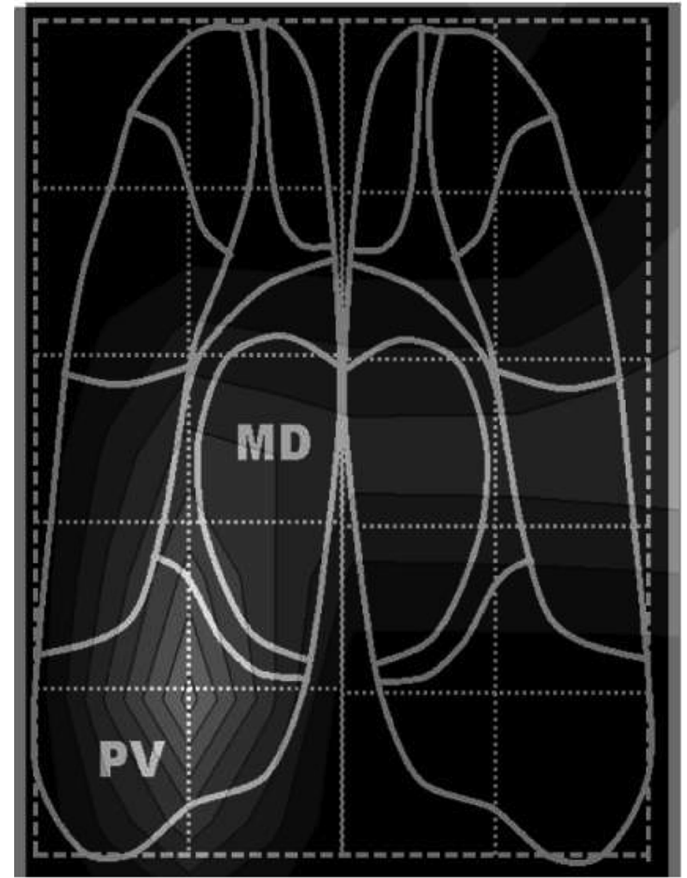

Fig. 4. Superimposition of contours from Fig. 2 and diagram of thalamus from Fig. 3, indicating mediodorsal nucleus (MD) and pulvinar (PV).

differences in glucose metabolism or BOLD activation in the mediodorsal, anterior, and pulvinar regions of the thalamus in patients with schizophrenia [6]-[9]. Other studies have reported schizophrenia-related reductions in volume and/or neuronal number in the thalamic nuclei highlighted by our techniques [11]-[16]. Altered glutamatergic NMDA receptor expression [17] and synaptic dysfunction or loss [18] have also been detected in the anterior and mediodorsal thalamic nuclei in patients with schizophrenia. Most importantly, our findings support and extend the results of previous spectroscopic investigations with voxels localized to the mediodorsal and anterior regions of the thalamus [26]-[28]. Those studies also found NAA deficits in patients with schizophrenia, but did not examine the pulvinar region.

Although the mediodorsal nucleus and pulvinar receive a wide range of inputs from other brain structures (limbic structures, basal ganglia, and brain stem nuclei), of particular interest when focusing on schizophrenia are the reciprocal connections of the thalamus with areas of cortex. All thalamic relays receive feedback from layer 6 of cortex. As well as receiving this feedback from cortex, "first-order relays" are those which send information to cortical areas about events happening in subcortical areas of the brain. In addition to receiving information from layer 6 of cortex, "higher order relays" receive additional input from layer 5 of cortex. In this way, higher order relays relay information already in cortex via driver input from layer 5 of one cortical area to middle areas of another cortical area [48]. The mediodorsal nucleus has the vast majority of its reciprocal higher order cortical connections with the prefrontal cortex [49], a part of the brain heavily involved in declarative memory. The highlighting of the mediodorsal nucleus by our ARD results is interesting when compared to the 
evidence that schizophrenic subjects show a deficit in working memory performance, as demonstrated by impairments in serial learning tasks and continuous performance tasks [50].

The pulvinar has extensive higher order connections to almost all visual cortical areas. The pulvinar may have a specific role in directing attention to stimuli in the visual field [51], and has been demonstrated to play a key role in pattern-motion selectivity and complex motion integration [52]. The highlighting of the pulvinar by our ARD results is interesting when compared to the evidence that schizophrenic subjects show deficits in such visual processing tasks [53]. The wider significance of our findings of reduced NAA in these areas of the thalamus is that they contribute to a growing body of work suggesting aberrant thalamic pathways in schizophrenia. By extending our analysis to include those brain regions connected to the pulvinar, and mediodorsal nucleus, we hope to gain further insight into this deficient thalamic circuitry.

Many diverse computational models of schizophrenia have been created, varying widely on the symptoms of schizophrenia modeled, the pathological factors assumed to cause the illness and the biological realism of the architecture of the computational model itself. Many models of schizophrenia have been proposed [54], [55] covering the effects of neuromodulators [56]. Some researchers have built computational models of the thalamus, cortex, or hippocampus, and investigated their abilities to model psychiatric disease. For example, Pelaez [57] investigated pattern completion in a neural network model of the thalamus and a biologically plausible model of synaptic plasticity in relation to schizophrenia, and it was proposed that the hallucinations observed were the result of aberrant pattern completion dynamics in thalamic areas. Work investigating the thalamus using a network of integrate-and-fire neurons to model analogical processing [58] suggests that the thalamic reticular nucleus may play a key role. Many cortical models of schizophrenia have used attractor-based models such as Hopfield [59] networks to generate models of dysfunction (where the central argument is that in schizophrenia too many, too few, or too strong attractors exist [60]-[74] Many other researchers have produced both evidence and models highlighting the importance of cortical dopaminergic mechanisms in schizophrenia [75]-[79] whereas related work [80] suggests that hyperconnectivity within a given cortical circuit leads to some of the manifestations of schizophrenia. Other models assume disruption of the dynamic connectivity between brain regions [81]-[85]. Various findings have emphasized hippocampal dysfunction [86]-[93]. There are many approaches to modeling schizophrenia described previously (for more in-depth reviews of these models, see [94] and [95]), while a host of other models are described in [96]-[100]. However, some current themes run through the majority of models.

- Autonomous output patterns that are pathological are formed by hyperassociation between memory circuits.

- The functional disconnection between different brain areas that are normally integrated is important (often hypothesized as decreased communication between frontal-hippocampal and/or cortical circuits).
However, although some of the aforementioned models attempt to take some inspiration from the gross structure of specific brain regions use biologically inspired learning rules (Hebbian-inspired rather than biologically unrealistic learning methods such as backpropagation) and attempt to relate to results generated by fMRI studies, none of the current models derive from neuroanatomically or neurochemically realistic data, such as that generated by recent studies using fMRI/MRSI and other analysis techniques. The models described previously have generated useful hypotheses about brain differences, and have been validated against behavioral data, but have not been tested using actual neurophysical data generated from schizophrenics and controls. Taking inspiration from these models, and the established connection between NAA and cognition, we intend to build a computational neuroscience model investigating the role of the thalamic structures outlined in the aforementioned research, and study its ability to replicate facets of the schizophrenic disease state. The model will test the effects of damage to the specific nuclei of the thalamus outlined in this paper, striatal hyper and frontal hypodopamine transmission, and NMDA receptor antagonism, with specific attention being paid to both first- and higher order relays and the thalamacortical loop. Such a model will be used to comment on the validity of previous approaches to cognitive modeling (encompassing models of both normal and abnormal cognition) and suggest new avenues for exploration in computational neuroscience.

\section{REFERENCES}

[1] S. Kapur, "Decoding schizophrenia-Putting the pieces together: Linking genes, neurochemistry, cognition, affect and neural networks,' 2005 [Online]. Available: http://www.omhf.on.ca/KapurAbstract.htm

[2] S. Frangou, X. Chitins, and S. C. Williams, "Mapping IQ and gray matter density in healthy young people," Neuroimage, vol. 23, no. 3, pp. 800-805, 2004.

[3] L. C. Konick and L. Friedman, "Meta-analysis of thalamic size in schizophrenia," Biol. Psychiatry, vol. 49, pp. 28-38, 2001.

[4] S. A. Mitelman, W. Byne, E. M. Kemether, E. A. Hazlett, and M. S. Buchsbaum, "Correlations between volumes of the pulvinar, centromedian, and mediodorsal nuclei and cortical Brodmann's areas in schizophrenia," Neurosci. Lett., vol. 392, pp. 16-21, 2006.

[5] J. D. Ragland, J. N. Valdez, J. Loughead, R. C. Gur, and R. E. Gur, "Functional magnetic resonance imaging of internal source monitoring in schizophrenia: Recognition with and without recollection," Schizophrenia Res., vol. 87, no. 1-3, pp. 160-171, 2006.

[6] E. A. Hazlett, M. S. Buchsbaum, E. Kemether, R. Bloom, J. Platholi, A. M. Brickman, L. Shihabuddin, C. Tang, and W. Byne, "Abnormal glucose metabolism in the mediodorsal nucleus of the thalamus in schizophrenia," Amer. J. Psychiatry, vol. 161, pp. 305-314, 2004.

[7] M. S. Buchsbaum, T. Someya, C. Y. Teng, L. Abel, S. Chin, A. Najafi, R. J. Haier, J. Wu, and W. E. Bunney, Jr., "PET and MRI of the thalamus in never-medicated patients with schizophrenia," Amer. J. Psychiatry, vol. 153, pp. 191-199, 1996.

[8] M. Katz, M. S. Buchsbaum, B. V. Siegel, Jr., J. Wub, R. J. Haier, and W. E. Bunney, Jr., "Correlational patterns of cerebral glucose metabolism in never-medicated schizophrenics," Neuropsychobiology, vol. 33, pp. $1-11,1996$

[9] J. Andrews, L. Wang, J. G. Csernansky, M. H. Gado, and D. Barch, "Abnormalities of thalamic activation and cognition in schizophrenia," Amer. J. Psychiatry, vol. 163, pp. 463-469, 2006.

[10] S. E. Rose, J. B. Chalk, A. L. Janke, M. W. Strudwick, L. C. Windus, D. E. Hannah, J. J. McGrath, C. Pantelis, S. J. Wood, and B. J. Mowry, "Evidence of altered prefrontal-thalamic circuitry in schizophrenia: An optimized diffusion MRI study,” NeuroImage, vol. 32, pp. 16-22, 2006. 
[11] G. J. Popken, W. E. Bunney, S. G. Potkin, and E. G. Jones, "Subnucleus-specific loss of neurons in medial thalamus of schizophrenics," Proc. Nat. Acad. Sci., vol. 97, pp. 9276-9280, 2000.

[12] K. A. Young, K. F. Manaye, C. Liang, P. B. Hicks, and D. C. German, "Reduced number of mediodorsal and anterior thalamic neurons in schizophrenia," Biol. Psychiatry, vol. 47, pp. 944-953, 2000.

[13] W. Byne, M. S. Buchsbaum, L. A. Mattiace, E. A. Hazlett, E. Kemether, S. L. Elhakem, D. P. Purohit, V. Haroutunian, and L. Jones, "Postmortem assessment of thalamic nuclear volumes in subjects with schizophrenia," Amer. J. Psychiatry, vol. 159, pp. 59-65, 2002.

[14] P. Danos, B. Baumann, A. Kramer, H.-G. Bernstein, R. Stauch, D. Krell, P. Falkai, and B. Bogerts, "Volumes of association thalamic nuclei in schizophrenia: A postmortem study," Schizophrenia Res., vol. 60, pp. 141-155, 2003.

[15] J. R. Highley, M. A. Walker, T. J. Crow, M. M. Esiri, and P. J. Harrison, "Low medial and lateral right pulvinar volumes in schizophrenia: A postmortem study," Amer. J. Psychiatry, vol. 160, 2003.

[16] A. R. Gilbert, D. R. Rosenberg, K. Harenski, S. Spencer, J. A. Sweeney, and M. S. Keshavan, "Thalamic volumes in patients with first-episode schizophrenia," Amer. J. Psychiatry, vol. 58, pp. 618-624, 2001.

[17] H. M. Ibrahim, A. J. Hogg, Jr., D. J. Healy, V. Haroutunian, K. L. Davis, and J. H. Meador-Woodruff, "Ionotropic glutamate receptor binding and subunit mRNA expression in thalamic nuclei in schizophrenia," Amer. J. Psychiatry, vol. 157, pp. 1811-1823, 2000.

[18] K. Blennow, N. Bogdanovic, M. Heilig, B. Grenfeldt, I. Karlsson, and P. Davidsson, "Reduction of the synaptic protein rab3a in the thalamus and connecting brain regions in post-mortem schizophrenic brains," $J$. Neural Transm., vol. 107, pp. 1085-1097, 2000.

[19] M. H. Baslow, "Evidence supporting a role for N-acetyl-L-aspartate as a molecular water pump in myelinated neurons in the central nervous system. An analytical review," Neurochem. Int., vol. 40, pp. 295-300, 2002.

[20] G. Chakraborty, P. Mekala, D. Yahya, G. Wu, and R. W. Ledeen, "Intraneuronal $\mathrm{N}$-acetylaspartate supplies acetyl groups for myelin lipid synthesis: Evidence for myelin-associated aspartoacylase," J. Neurochem., vol. 78, pp. 736-745, 2001.

[21] G. Tsai and J. Coyle, "N-acetylaspartate in neuropsychiatric disorders," Progr. Neurobiol., vol. 46, pp. 531-540, 1995.

[22] D. L. Arnold, N. deStefano, P. M. Matthews, and B. D. Trapp, "N-acetylaspartate: Usefulness as an indicator of viable neuronal tissue," Ann. Neurol., vol. 50, pp. 823-825, 2001.

[23] R. E. Jung, R. A. Yeo, S. J. Chiulli, J. Stephen, L. Wilmer, Jr., D. C. Weers, B. L. Blaine, and W. M. Brooks, "Biochemical markers of cognition: A proton MR spectroscopy study of normal human brain," Neuroreport, vol. 10, no. 16, pp. 3327-3331, 1999.

[24] R. E. Jung, R. A. Yeo, S. J. Chiulli, W. L. Sibbitt, and W. M. Brooks, "Myths of neuropsychology: Intelligence, neurometabolism and cognitive ability," Clin. Neuropsychologist, vol. 14, pp. 535-545, 2000.

[25] D. P. Auer, M. Wilke, A. Grabner, J. O. Heidenreich, T. Bronisch, and T. C. Wetter, "Reduced NAA in the thalamus and altered membrane and glial metabolism in schizophrenic patients detected by $1 \mathrm{H}-\mathrm{MRS}$ and tissue segmentation," Schizophrenia Res., vol. 52, pp. 87-99, 2001.

[26] R. F. Deicken, C. Johnson, Y. Eliaz, and N. Schuff, "Reduced concentrations of thalamic $\mathrm{N}$-acetylaspartate in male patients with schizophrenia," Amer. J. Psychiatry, vol. 157, pp. 644-647, 2000.

[27] G. Ende, D. F. Braus, S. Walter, and F. A. Henn, "Lower concentration of thalamic N-acetylaspartate in patients with schizophrenia: A replication study," Amer. J. Psychiatry, vol. 158, pp. 1314-1316, 2001.

[28] A. Jakary, S. Vinogradov, R. Feiwell, and R. F. Deicken, "N-acetylaspartate reductions in the mediodorsal and anterior thalamus in men with schizophrenia verified by tissue volume corrected proton MRSI," Schizophrenia Res., vol. 76, no. 2-3, pp. 173-185, 2005.

[29] H. Hagino, M. Suzuki, K. Mori, S. Nohara, I. Yamashita, T. Takahashi, K. Kurokawa, M. Matsui, N. Watanabe, H. Seto, and M. Kurachi, "Proton magnetic resonance spectroscopy of the inferior frontal gyrus and thalamus and its relationship to verbal learning task performance in patients with schizophrenia: A preliminary report," Psychiatry Clin. Neurosci., vol. 56, pp. 499-507, 2002.

[30] J. Theberge, Y. Al-Semaan, P. C. Williamson, R. S. Menon, R. W. J. Neufeld, N. Rajakumar, B. Schaefer, M. Densmore, and D. J. Drost, "Glutamate and glutamine in the anterior cingulate and thalamus of medicated patients with chronic schizophrenia and healthy comparison subjects measured with 4.0-T proton MRS," Amer. J. Psychiatry, vol. 160, pp. 2231-2233, 2003.

[31] A. Bertolino, "Regionally specific neuronal pathology in untreated patients with schizophrenia: A proton magnetic resonance spectroscopic imaging study," Biol. Psychiatry, vol. 43, pp. 641-648, 1998.
[32] A. Browne, B. D. Hudson, D. C. Whitley, M. G. Ford, and P. Picton, "Biological data mining with neural networks: Implementation and application of a flexible decision tree extraction algorithm to genomic problem domains," Neurocomputing, vol. 57, pp. 275-293, 2004.

[33] I. Guyon and A. Elissee, "Special issue on variable and feature selection,” J. Mach. Learn. Res. 2003 [Online]. Available: http://www.jmlr. org/papers/special/feature.html

[34] D. J. C. MacKay, "A practical Bayesian framework for backpropagation networks," Neural Comput., vol. 4, no. 3, pp. 448-472, 1992.

[35] R. Neal, "Bayesian learning for neural networks," Ph.D. dissertation, Dept. Comput. Sci., Univ. Toronto, Toronto, ON, Canada, 1994.

[36] H. H. Thodberg, "A review of Bayesian neural networks with an application to near-Infrared spectroscopy," IEEE Trans. Neural Netw., vol. 7, no. 1, pp. 56-72, Jan. 1996.

[37] I. Ulusoy and C. M. Bishop, "Automatic relevance determination for the estimation of relevant features for object recognition," in Proc. 14th Conf. Signal Process. Commun. Appl., 2006, pp. 1-4.

[38] M. E. Tipping, "Bayesian inference: An introduction to principles and practice in machine learning," in Advanced Lectures on Machine Learning. New York: Springer-Verlag, 2004, pp. 41-62.

[39] I. Nabney, NETLAB: Algorithms for Pattern Recognition. New York: Springer-Verlag, 2004.

[40] I. Nabney, NETLAB [Online]. Available: http://www.ncrg.aston.ac.uk/ netlab

[41] R. F. Deicken, Y. Eliaz, R. Feiwell, and N. Schuff, "Increased thalamic $\mathrm{N}$-acetylaspartate in male patients with familial bipolar I disorder," Psychiatry Res. Neuroimaging, vol. 106, pp. 35-45, 2001.

[42] M. F. A. Moller, "A scaled conjugate gradients algorithm for fast supervised learning," Neural Netw., vol. 6, no. 4, pp. 525-533, 1993.

[43] S. J. Hanson and Y. Halchenko, "Brain reading using full brain Support Vector Machines for object recognition: There is no face identification area," Neural Comput., vol. 20, pp. 486-503, Feb. 2008.

[44] V. N. Vapnik, The Nature of Statistical Learning Theory. New York: Springer-Verlag, 1995.

[45] T. VanGestel, J. A. K. Suykens, B. DeMoor, and J. Vandewalle, "Automatic relevance determination for least squares support vector machines," in Proc. IEEE Int. Joint Conf. Neural Netw., 2001, vol. 4, pp. 2416-2421.

[46] C. Gold, A. Holub, and P. Sollich, "Bayesian approach to feature selection and parameter tuning for support vector machine classifiers," Neural Netw., vol. 18, no. 5-6, pp. 693-701, 2005.

[47] S. J. Hanson, personal communication.

[48] S. M. Sherman and R. W. Guillery, Exploring the Thalamus and Its Role in Cortical Function. Cambridge, MA: MIT Press, 2006.

[49] Y. VanDerWerf, J. Jolles, M. P. Witter, and H. B. M. Uylings, "Contributions of thalamic nuclei to declarative memory functioning," Cortex, vol. 39, pp. 1047-1062, 2003.

[50] S. A. Mitelman, W. Byne, E. M. Kemether, E. A. Hazlett, and M. S. Buchsbaum, "Metabolic disconnection between the mediodorsal nucleus of the thalamus and cortical Brodmann's area of the left hemisphere in schizophrenia," Amer. J. Psychiatry, vol. 162, no. 9, pp. 1733-1735, 2005.

[51] D. LaBerge, "Attention, awareness and the triangular circuit," Consciousness Cogn., vol. 6, pp. 149-181, 1997.

[52] M. Y. Villeneuve, R. Kupers, A. Gjedde, M. Ptito, and C. Casanova, "Pattern-motion selectivity in the human pulvinar," Neuroimage, vol. 28, pp. 474-480, 2005.

[53] S. B. Hutton and U. Ettinger, "the antisaccade task as a research tool in psychopathology: A critical review," Psychophysiology, vol. 43, pp. 302-313, 2006.

[54] G. S. Grossberg and J. Pepe, "Schizophrenia: Possible dependence of associational span, bowing, and primacy vs. recency on spiking threshold," Behavioral Sci., vol. 15, no. 4, pp. 359-362, 1970.

[55] S. Grossberg, "The imbalanced brain: From normal behavior to schizophrenia," Biol. Psychiatry, vol. 48, no. 2, pp. 81-98, 2000.

[56] D. Hestenes, "Modulatory mechanisms in mental disorders," in Neural Networks and Psychopathology, D. Stein and J. Ludick, Eds. Cambridge, U.K.: Cambridge Univ. Press, 1998, pp. 132-164.

[57] J. R. Pelaez, "Towards a neural network based therapy for hallucinatory disorders," Neural Netw., vol. 13, no. 8-9, pp. 1047-1061, 2000.

[58] Y. Choe, "The role of temporal parameters in a thalamocortical model of analogy," IEEE Trans. Neural Netw., vol. 15, no. 5, pp. 1071-82, Sep. 2004.

[59] J. J. Hopfield and D. W. Tank, "Neural computation of decisions in optimization problems," Biol. Cybern., vol. 52, pp. 144-152, 1985.

[60] G. G. Globus and J. P. Arpaia, "Psychiatry and the new dynamics," Biol. Psychiatry, vol. 35, pp. 352-364, 1994. 
[61] R. E. Hoffman, "Computer simulations of neural information processing and the schizophrenia-mania dichotomy," Arch. Gen. Psychiatry, vol. 44, pp. 178-188, 1987.

[62] R. E. Hoffman, J. Rapaport, C. M. Mazure, and D. M. Quinlan, "Selective speech perception alterations in schizophrenia patients reporting hallucinated voices," Amer. J. Psychiatry, vol. 156, pp. 393-399, 1999.

[63] R. E. Hoffman, J. Glist, C. M. Mazure, and D. M. Quinlan, "Nonlanguage cognitive deficits and hallucinations in schizophrenia: Reply," Amer. J. Psychiatry, vol. 157, pp. 487-488, 2000.

[64] T. H. Jobe, R. Vimal, A. Kovilparambil, J. D. Port, and M. M. Gaviria, "A theory of cooperativity modulation in neural networks as an important parameter of CNS catecholamine function and induction of psychopathology," Neurol. Res., vol. 16, no. 5, pp. 330-41, 1994.

[65] T. H. Jobe, C. G. Fichtner, J. D. Port, and M. M. Gaviria, "Neuropoiesis: Proposal for a connectionist neurobiology," Med. Hypotheses, vol. 45, no. 2, pp. 147-63, 1995.

[66] T. H. Jobe and M. M. Gaviria, "Computer simulations of neural information processing and the schizophrenia-mania dichotomy: Commentary," J. Psychosom. Res., vol. 44, pp. 629-630, 1998.

[67] E. Ruppin, J. Reggia, and D. Horn, "Pathogenesis of schizophrenic delusions and hallucinations: A neural model of positive schizophrenic symptoms," Schizophrenia Bull., vol. 22, no. 1, pp. 105-123, 1996.

[68] D. Horn and E. Ruppin, "Compensatory mechanisms in an attractor neural network model of schizophrenia," Neural Comput., vol. 7, pp. 182-205, 1995.

[69] A. Greenstein-Messica and E. Ruppin, "Synaptic runaway in associative networks and the pathogenesis of schizophrenic psychosis," Neural Comput., vol. 10, no. 2, pp. 451-465, 1998.

[70] E. Ruppin, "NMDA delayed maturation and schizophrenia," Med. Hypotheses, vol. 54, no. 5, pp. 693-697, 2000.

[71] S. Heckers, S. L. Rauch, D. Goff, C. R. Savage, D. L. Schacter, A. J. Fischman, and N. M. Alpert, "Impaired recruitment of the hippocampus during conscious recollection in schizophrenia," Nature Neurosci., vol. 1, no. 4, pp. 318-323, 1998

[72] M. H. Joseph, C. D. Frith, and J. L. Waddington, "Dopaminergic mechanisms and cognitive deficit in schizophrenia," Psychopharmacology, vol. 63, no. 3, pp. 273-280, 1979.

[73] J. D. Cohen, T. S. Braver, and R. C. OReilly, "A computational approach to prefrontal cortex, cognitive control, and schizophrenia: Recent developments and current challenges," Philosoph. Trans. Roy. Soc. Lond. B, vol. 351, pp. 1515-1527, 1996.

[74] G. Siegle, M. Harrow, J. Sands, A. Miller, and T. Jobe, "Does stimulus difficulty play a role in thought pathology? Schizophrenics vs. Depressives," in Proc. Soc. Res. Psychopathol., Chicago, IL, 1993.

[75] T. S. Braver, D. M. Barch, and J. D. Cohen, "Cognition and control in schizophrenia: A computational model of dopamine and prefrontal function," Biol. Psychiatry, vol. 46, pp. 312-328, 1999.

[76] A. Reid and D. Willshaw, "Modeling prefrontal cortex delay cells: The role of dopamine in schizophrenia," in Disorders of Brain, Behavior, and Cognition: The Neurocomputational Perspective, J. A. Reggia, E. Ruppin, and D. L. Glanzman, Eds. New York: Elsevier, 1999, pp. 351-374.

[77] D. Servan-Schreiber and J. D. Cohen, "Dopamine, frontal cortex, and schizophrenia: Model and data," in Neural Modeling of Brain and Cognitive Disorders, J. Reggia, E. Ruppin, and R. Berndt, Eds. Singapore: World Scientific, 1996.

[78] D. Servan-Schreiber and J. D. Cohen, "Stroop task, language, and neuromodulation: Models of cognitive deficits in schizophrenia," in Fundamentals of Neural Network Modeling: Neuropsychology and Cognitive Neuroscience, R. W. Parks, D. S. Levine, and D. L. Long, Eds. Cambridge, MA: MIT Press, 1998, pp. 192-208.

[79] A. Roelofs, "Goal-referenced selection of verbal action: Modeling of attentional control in the Stroop task," Psychol. Rev., vol. 110, pp. $88-125,2003$.

[80] S. Vinogradov, J. H. Poole, and J. Willis-Shore, "Produced by either God or Satan: Neural network approaches to delusional thinking," in Neural Networks and Psychopathology, D. Stein and J. Ludick, Eds. Cambridge, U.K.: Cambridge Univ. Press, 1998, pp. 189-231.

[81] N. C. Andreasen, "A unitary model of schizophrenia: Bleulers fragmented phrene as schizencephaly," Arch. Gen. Psychol., vol. 56, pp. 781-787, 1999.

[82] A. Peled, "Multiple constraint organization in the brain: A theory for schizophrenia," Brain Res. Bull., vol. 49, pp. 245-250, 1999.

[83] G. Tononi and G. M. Edelman, "Schizophrenia and the mechanisms of conscious integration,” Brain Res. Rev., vol. 31, pp. 391-400, 2000.
[84] K. J. Friston, "The labile brain. I. Neuronal transients and nonlinear coupling," Philos. Trans. Roy. Soc.. Lond. B, vol. 355, pp. 215-236, 2001.

[85] M. Breakspear, J. R. Terry, K. J. Friston, A. W. Harris, L. M. Williams, K. Brown, J. Brennan, and E. Gordon, "A disturbance of nonlinear interdependence in scalp EEG of subjects with first episode schizophrenia," NeuroImage, vol. 20, pp. 466-478, 2003.

[86] E. T. Rolls, "Functions of neuronal networks in the hippocampus and neocortex in memory," in Neural Models of Plasticity: Experimental and Theoretical Approaches, J. H. Byrne and W. O. Berry, Eds. San Francisco, CA: Academic, 1989, pp. 240-265.

[87] E. Y. H. Chen, "A neural network model of cortical information processing in schizophrenia I: Interaction between biological and social factors in symptom formation," Can. J. Psychiatry, vol. 39, pp. 362-367, 1994.

[88] E. Y. H. Chen, A Neurocomputational Model of Early Psychosis, ser. Lecture Notes in Computer Science 2774, V. Palade, R. J. Howlett, and L. C. Jain, Eds. Berlin, Germany: Springer-Verlag, 2003, pp. $1149-1155$

[89] P. J. Harrison, "The neuropathology of schizophrenia: A critical review of the data and their interpretation," Brain, vol. 122, pp. 593-624, 1999.

[90] S. E. Arnold, "Cellular and molecular neuropathology of the parahippocampal region in schizophrenia," Ann. NY Acad. Sci., vol. 911, pp. $275-292,2000$

[91] I. C. Wright, "Meta-analysis of regional brain volumes in schizophrenia," Amer. J. Psychiatry, vol. 157, pp. 16-25, 2000.

[92] M. Meeter, J. M. J. Murre, and L. M. Talamini, "A computational approach to memory deficits in schizophrenia," Neurocomputing, vol. 44, pp. 929-936, 2002.

[93] M. Meeter, L. M. Talamini, J. M. J. Murre, B. Elvevag, and T. E. Goldberg, "Integration in the parahippocampal gyrus: A computational memory model with implications for schizophrenia," presented at the Meeting Cogn. Sci. Soc., NY, 2003.

[94] G. J. Siegle, "Connectionist models of cognitive, affective, brain and behavioral disorders," Tech. Rep., 1998 [Online]. Available: http://www.sci.sdsu.edu/CAL/connectionist-models

[95] L. Aakerlund and R. Hemmingsen, "Neural networks as models of psychopathology," Biol. Psychiatry, vol. 43, pp. 471-482, 1998.

[96] M. Spitzer, "A cognitive neuroscience view of schizophrenic thought disorder," Schizophrenia Bull., vol. 23, pp. 29-50, 1997.

[97] J. RoperoPelez, "Towards a neural network based therapy for hallucinatory disorders," Neural Netw., vol. 13, pp. 1047-1061, 2000.

[98] G. V. Wallenstein and M. E. Hasselmo, "GABAergic modulation of hippocampal population activity: Sequence learning, place field development, and the phase precession effect," J. Neurophysiol., vol. 78, pp. 393-408, 1997.

[99] M. E. Hasselmo, J. Hay, M. Ilyn, and A. Gorchetchnikov, "Neuromodulation, theta rhythm and rat spatial navigation," Neural Netw., vol. 15, pp. 689-707, 2002.

[100] B. P. Graham, "Dynamics of storage and recall in hippocampal associative memory networks," in Computational Neuroscience: Cortical Dynamics, ser. Lecture Notes in Computer Science 3146, P. Erdi, A. Esposito, M. Marinaro, and S. Scarpetta, Eds. Berlin, Germany: Springer-Verlag, 2004, pp. 1-23.

Antony Browne, photograph and biography not available at the time of publication.

Angela Jakary, photograph and biography not available at the time of publication.

Sophia Vinogradov, photograph and biography not available at the time of publication.

Yu Fu, photograph and biography not available at the time of publication.

Raymond F. Deicken, photograph and biography not available at the time of publication. 